Revista Iberoamericana, Vol. LXXVI, Núm. 231, Abril-Junio 2010, 409-424

\title{
LA BÚSQUEDA DE LA JUSTICIA SOCIAL: LA LUCHA CONTRA LA HOMOFOBIA Y EL RACISMO EN LA NOVELA NEGRA DE MICHAEL NAVA
}

\author{
POR \\ DANIEl ENRIQUe PÉREZ \\ University of Nevada, Reno
}

Entre los escritores chicanos y latinos de la novela negra en los Estados Unidos, ninguno ha tenido tanto éxito como el escritor chicano Michael Nava. De hecho, Nava se ha convertido en el autor latino más renombrado y más publicado de la novela negra en los EE.UU. La serie que lo estableció como escritor de mayor importancia consiste en siete novelas, abarca un período de quince años (19862001) y narra la vida y las aventuras de un detective chicano que también es gay, Henry Rios (sic). El hecho de que la serie haya tenido tanto éxito y que Nava sea un autor gay chicano que ubica en el centro de sus novelas a un personaje principal gay chicano, es un fenómeno sin igual en los EE.UU. Además, Nava trata el tema de la homosexualidad abiertamente en cada novela de la serie y es evidente que el autor, tanto como el protagonista principal, siente un compromiso social, en cuanto al hecho de combatir la homofobia. El autor utiliza su experiencia como abogado para crear una narrativa que subraya las injusticias que sufren los hombres gay y las lesbianas. Sin embargo, el protagonista, Henry Rios, experimenta algunos conflictos de identidad precisamente por ser chicano y por ser gay. Este ensayo demostrará la manera en que Nava presenta y trata los conflictos de identidad que surgen a lo largo de la serie. Tales conflictos se basan en dos de los factores más sobresalientes de la identidad de Henry Rios: su sexualidad y su herencia cultural. Como abogado gay y chicano, Rios vive una vida sumamente marginada. Para él, entre los desafíos numerosos que precisa superar, figura el de crear un espacio seguro y cómodo para él mismo dentro de un mundo dominado por lo anglosajón y la homofobia. Como víctima del neocolonialismo, Rios se convierte en un ejemplo contemporáneo del sujeto colonizado.

Para evaluar el desarrollo de estos dos factores y el impacto que tienen sobre el desarrollo de la identidad del protagonista principal a lo largo de la serie, se puede hacer un estudio comparativo entre la primera novela de la serie, The Little Death (1986) y la última novela, Rag and Bone (2001). Al emprender este tipo de estudio, 
es posible evaluar la evolución de Rios en cuanto a su lucha contra la homofobia y el racismo. Este estudio demostrará que mientras Rios batalla contra la homofobia y el racismo que observa y experimenta en la sociedad, a la vez es víctima de un conflicto interno de identidad que se puede caracterizar por los mismos elementos. Durante el transcurso de la serie, Rios se enfrenta con varios problemas personales: lucha contra el alcoholismo, varias relaciones fracasadas, la muerte de un amante que padecía SIDA y la realidad de vivir en una sociedad homofóbica y racista. De interés particular es la manera en que Rios maneja sus conflictos internos de identidad, los cuales funcionan en forma paralela a los conflictos externos que conscientemente intenta combatir.

$\mathrm{Al}$ hacer un análisis de la primera novela, The Little Death, se puede ver el planteamiento de las raíces de los dos compromisos del autor que dirigirán la narrativa a lo largo de la serie: su interés en destacar y combatir las injusticias de un ambiente homofóbico y superar el conflicto de identidad que experimenta por ser chicano. Por lo tanto, la sexualidad y la herencia cultural son los dos factores más importantes en el desarrollo del personaje Henry Rios, los dos afectan sus interacciones diarias con sus colegas y sus clientes y los dos tienen una influencia preponderante sobre la manera en que resuelve los casos misteriosos que se presentan. En esta novela, Nava nos presenta a Henry Rios, un abogado joven (tiene más o menos treinta años) que defiende a los criminales. Aunque la primera novela da muy pocas pistas en cuanto a la herencia cultural de Rios, una mirada cuidadosa revela que la identidad étnica del protagonista no puede ser pasada por alto. De hecho, sugeriría que la etnia de Rios es parte del misterio que se debe resolver. Hay algunos signos de que Rios puede ser un personaje chicano o latino, específicamente, su apellido, que, aunque se escribe sin acento, es, obviamente, de origen español. La yuxtaposición de un nombre en inglés, Henry (cuyo equivalente en español sería Enrique), y Rios es la única pista que le da al lector una idea de la herencia cultural del personaje. Este misterio sí se resuelve durante el transcurso de la serie, pero el hecho de que sea un misterio, especialmente al principio, provoca una indagación acerca de la identidad cultural del protagonista.

No existen otras referencias a la herencia cultural de Rios en The Little Death. El personaje usa un inglés estándar y pulido; las únicas palabras en español en toda la novela son los nombres de las calles o las ciudades en California: San Francisco, San Jose, San Joaquin -nunca acentuadas, por supuesto. Ahora bien, el uso del lenguaje no necesariamente constituye una herencia específica. La falta de los acentos en las palabras españolas se puede justificar por el hecho de que la novela está escrita en inglés. No obstante, en una novela donde no existen otras señales, el nombre se convierte en una característica especial que se puede usar para hacer conclusiones sobre el origen del personaje. Además del personaje principal, sólo

Revista Iberoamericana, Vol. LXXVI, Núm. 231, Abril-Junio 2010, 409-424
ISSN 2154-4794 (Electrónico) 
hay un personaje más que posiblemente es chicano o latino, Samuel Torres, un detective que, con respecto a su herencia cultural, también exhibe una identidad tan ambigua como la de Rios.

Por lo tanto, la novela se centra en el asesinato de Hugh Paris, un hombre gay anglosajón que es de una familia poderosa y adinerada. En esta novela, Nava decide enfocarse en la sexualidad de sus protagonistas principales en lugar de la herencia cultural. No obstante, creo que precisamente por esta razón la cuestión sobre la herencia cultural de los protagonistas es tan interesante. Por ejemplo, cuando le preguntan a Rios si su papá también era abogado, responde, "No, he was foreman of the night crew at a cannery in Marysville. I'm the only lawyer in my family" (91). Esta es la única referencia que se hace con respecto a su familia y, aunque significa que viene de una familia obrera y sugiere cierta perspectiva ideológica, no indica necesariamente que es de una familia chicana ni mexicana. Rios está completamente sumergido en la cultura anglosajona. De hecho, yo sugerería que no se puede verificar que la novela tenga algún personaje definitivamente chicano o latino. Henry Rios no tiene ninguna característica distintiva que lo pueda definir como chicano o como latino. Hasta su nombre indica que no se ubica dentro de este espacio cultural. La decisión de Nava de omitir cualquier referencia a la identidad del protagonista se puede entender como una manera de demostrar que el protagonista está sufriendo de algún conflicto de identidad. En la novela, hay varias indicaciones que Rios padece de un complejo de inferioridad. Por ejemplo, sólo tiene relaciones sexuales con hombres anglosajones, típicamente, con pelo rubio y ojos azules. En la novela, dichos hombres se presentan como íconos de la belleza ideal masculina o del clon gay macho. ${ }^{1}$ De alguna manera, Rios es un anti-héroe; padece de un complejo de inferioridad, es alcohólico y está tan desilusionado con su profesión como abogado que apenas se puede animar a trabajar. Al principio, ni siquiera tiene la energía para investigar el crimen de Hugh Paris. El ímpetu que lo mueve es que está interesado en Paris erótica y emocionalmente. En la escena que sigue, Paris ha sido arrestado por cuestiones de uso de drogas y pasa una noche en la cárcel. Rios lo ve por primera vez en la mañana y lo describe como un adonis:

I read the report. The suspect's name was Hugh Paris. He stood five-foot ten, had blond hair and blue eyes. He refused to give an address or answer questions about his employment or his family. He had no criminal record. I studied his booking photo. His hair was in his face and his eyes went off in two different directions, but there was no denying he was an exceptionally handsome man. (11)

\footnotetext{
1 A este respecto véase el estudio de Michael P. Levine, Gay Macho, para un discurso sobre el clon gay macho.

ISSN 0034-9631 (Impreso)
} 
En otra escena, Rios intenta visitar a su amigo y colega, Aaron Gold, pero Gold acaba de ser asesinado. Rios tiene una confrontación, en la oscuridad, con el asesino, a quien Rios logra quitarle su pistola. El asesino se escapa y Rios se queda con la pistola en su mano. Cuando llega la policía, Rios es erróneamente sospechado de haber cometido el crimen y es arrestado. El abogado de Rios, Scott Patterson, aclara la situación. Resulta que un vecino vio al asesino entrar en la casa de Gold y le dio una descripción del criminal verdadero. El abogado Patterson le explica a Rios, "Well [the neighbor], saw a guy. Blond, about your height. Good build. Good looking. Couldn’t be you” (131). Irónicamente, ésta es la mejor descripción de Rios en toda la novela -una imagen que sólo se puede formar por medio de contemplar la antítesis de la descipción del asesino verdadero. El comentario que hace Patterson se puede interpretar de varias maneras. Primero, Rios no puede ser el asesino o porque no tiene pelo rubio, o porque no está de buena forma físicamente (aunque sí se sabe que él corre y hace ejercicios), o porque no es atractivo. Sin embargo, existe la posibilidad de que Patterson le esté diciendo que no puede ser el asesino porque no es atractivo y no puede ser atractivo porque no tiene pelo rubio. No obstante, lo que es importante aquí, y en el resto de la novela, es que el autor constantemente representa la belleza ideal con características distintivamente anglosajonas que no permite la construcción de otros modelos de belleza. En su estudio sobre la estética del color blanco, White (1997), Richard Dyer describe la formación y el establecimiento de la noción del color blanco como característica obligatoria de la belleza ideal. Dyer hace una conexión entre esta noción de lo blanco como ideal y el cuerpo masculino. Sugiere que el intento de formar un cuerpo musculoso es un intento de llegar hasta un nivel de belleza que se basa en la estética del cuerpo anglosajón masculino: "Whiteness [...] is an aspirational structure, requiring ideals of human development. All the rhetoric of body-building is founded on this and most vividly seen in the aspirational motifs of the posing vocabulary, bodies forever striving upwards" (151-2).

Ahora bien, Rios no se percibe como un hombre atractivo y, en su mayor parte, los personajes secundarios de The Little Death tampoco lo perciben de esa manera. Por falta de rasgos anglosajones, Rios queda al margen del discurso sobre la belleza masculina; como no le es permitido participar en tal discurso, Nava lo ofrece como víctima de una sociedad racista. Además, Rios ha interiorizado y aceptado que es inferior no sólo por el hecho de no ser anglosajón, sino también por ser gay. Simbólicamente, la herencia cultural de Rios ha sido enterrada y, según Frantz Fanon, cuando esto le ocurre a un sujeto colonizado, se desarrolla un complejo de inferioridad:

Every colonized people-in other words, every people in whose soul an inferiority complex has been created by the death and burial of its local cultural originality-

Revista Iberoamericana, Vol. LXXVI, Núm. 231, Abril-Junio 2010, 409-424
ISSN 2154-4794 (Electrónico) 
finds itself face to face with the language of the civilizing nation; that is, with the culture of the mother country. The colonized is elevated above his jungle status in proportion to his adoption of the mother country's cultural standards. He becomes whiter as he renounces his blackness, his jungle. (18)

Dicho complejo de inferioridad parece tener sus raíces en los dos temas que estoy explorando en este ensayo: la homofobia y el racismo. Propongo que el racismo que moldea al protagonista es un racismo interno porque no existe mucha evidencia de que Rios sea víctima de fuerzas exteriores (un racismo institucionalizado), pero sí hay mucha evidencia de que se enfrenta con la homofobia (interna y externa) diariamente. Rios, entonces, exhibe una tendencia a reprimir las señas de su identidad cultural, ejemplificando, así, lo que Anne Stoler destaca sobre el racismo en Race and the Education of Desire (1995), donde lo que se reprime de una herencia cultural es un producto directo de la internalización del racismo.

No obstante, en The Little Death, es evidente que el mayor interés de Rios es luchar contra la homofobia. La novela se abre con una escena en la que Rios está en la oficina revisando los casos de los criminales que fueron arrestados durante la noche. Después de que el ayudante del sheriff describe el caso de Hugh Paris, le comenta a Rios, "[he's in] the drunk tank with the queens. He's a fag” (11). Inmediatamente, Rios responde, "That's no crime” (11). Mientras lucha abiertamente contra una sociedad homofóbica, Rios también lidia con su homofobia interna e interiorizada. Ahora bien, Rios vive en un espacio donde la visibilidad de lo queer es más común que en otras partes de los EE.UU., San Francisco. ${ }^{2}$ Por lo tanto, tiene el privilegio de poder estar en un espacio donde se encuentran muchas personas gay y donde no existen tantas amenazas homofóbicas. No obstante, Rios no se permite ser relegado sólo a estos espacios. Cuando él y Paris empiezan una relación íntima, se encuentran en el distrito Castro de San Francisco-conocido universalmente como un espacio cultural gay. Mientras caminan en el centro del distrito, Paris toma la mano de Rios en la suya y siguen por la calle. Para Rios, este acto es un poco raro: "It perplexed me how sex with other men seemed natural to me but not the small physical gestures of affection and concern” (44). A pesar de esta incomodidad, Rios sabe que necesita acostumbrarse a vivir abiertamente como hombre gay. Cuando la pareja sale del borde entre la zona Castro y el resto de San Francisco, los dos están muy conscientes de que ahora no están protegidos por las normas del espacio de donde acaban de salir. Paris abre su mano e intenta abrir un espacio entre ellos pero, en un momento elocuente de la novela, Rios toma la mano de Paris y los dos

2 Véase el estudio de Michelangelo Signorile, Life Outside, para un discurso sobre la dinámica de los espacios urbanos gay de los EE.UU.

ISSN 0034-9631 (Impreso) 
siguen caminando, listos para enfrentarse con una sociedad homofóbica: “[Hugh] looked at me, startled, then tightened his grip. And life went on” (44).

En sus apreciaciones sobre el personaje de Nava, David William Foster propone que la homofobia es la fuerza principal que mantiene a Rios al margen de la sociedad. Para Foster, la homofobia sirve para excluir y expulsar a Rios de la sociedad. Sugiere que experimenta una forma de "ninguneo" que procede de su abyección extrema: no sigue las normas del sistema jurídico donde trabaja, no gana mucho dinero y vive en una casa sencilla y desordenada que se ubica fuera del centro de la ciudad (81). Consiguientemente, Rios se esfuerza por separarse de las instituciones dominadas por la cultura anglosajona porque no se encuentra capaz de identificarse con ese mundo. De hecho, siempre hace comparaciones entre su historia como miembro de la clase obrera y el mundo del anglosajón de la clase alta. El contraste entre las dos posiciones lo marca como sujeto abyecto, al margen de la sociedad por su etnicidad, su orientación sexual y su clase social. Por ejemplo, en The Little Death, después de describir a un amigo que conoció cuando estaba en la Facultad de Derecho, Grant Hancock, un varón de familia adinerada, Rios revela la distancia y la marginalidad que marca su existencia, "In the normal course of existence, I would never have met someone like Grant since his world was far removed from mine” (70).

La noción de diferencia que establece Rios procede de una combinación de características personales: su sexualidad, su pobreza y su herencia cultural. Como ha insistido Jacques Lacan, el desarrollo del individuo se hace por el reconocimiento de la diferencia entre el sujeto y el "Otro". Además, en su intento de llegar al centro de un universo simbólico donde existe la posibilidad de ser reconocido, el sujeto siempre tiene el deseo de ser el "Otro": "In short, nowhere does it appear more clearly that man's desire finds its meaning in the desire of the other, not so much because the other holds the key to the object desired, as because the first object of desire is to be recognized by the other" (58). Por lo tanto, los tres aspectos de identidad subrayados arriba contribuyen a la formación de un discurso sobre el conflicto de identidad interno que experimenta el protagonista.

Mientras que The Little Death tiene muy pocas referencias a la familia del protagonista principal, ya en la tercera novela de la serie, How Town (1990), Nava presenta a Elena -la hermana lesbiana de Rios. Después de muchos años sin comunicarse los dos, Elena entra en la vida del protagonista principal y Rios es forzado a revivir su traumática adolescencia, la cual fue atormentada por su padre mexicano que solía ser violento y homofóbico. Poco después, las tareas diarias hacen que Rios reflexione sobre su niñez. Según Foster, en el análisis que hace de The Burning Plain, la sexta novela de la serie, cuando Rios es vícitima de la homofobia (emocional o físicamente), se acuerda del abuso de su papá. Además, Foster sugiere

\footnotetext{
Revista Iberoamericana, Vol. LXXVI, Núm. 231, Abril-Junio 2010, 409-424 ISSN 0034-9631 (Impreso) ISSN 2154-4794 (Electrónico)
} 
que Rios se da cuenta de que su padre estaba actuando dentro de las normas de un discurso universal que esboza el machismo y facilita la homofobia (76).

Por lo tanto, la homofobia experimentada a manos de su propio padre traumatiza al Rios adulto, tanto como cuando era adolescente. Al salirse de la casa/el barrio donde se crió y de acomodarse en las instituciones académicas y profesionales, Rios logra escaparse físicamente del abuso de su papá. No obstante, internamente sigue viviendo con las heridas emocionales. La herencia cultural mexicana que estoy examinando es uno de los elementos que conforman el complejo de inferioridad por el hecho de que la fuente de tal abuso es su padre. Sugiero que Rios rechaza su herencia cultural y se acomoda en un mundo anglosajón porque su historia está marcada por varios factores negativos: la violencia, la pobreza, la homofobia y el racismo. Por eso, Rios persigue al "Otro" descrito por Lacan, siempre añorando reconocimiento y viviendo obsesionado con la belleza del hombre anglosajón. Sin embargo, es notable que este fenómeno no continúa a lo largo de la serie. Durante el transcurso de la serie, Rios llega a controlar estos conflictos de identidad y logra crear un espacio interno donde ya no se siente víctima de la homofobia ni del racismo. Es decir, el nivel de la tensión que cada elemento proporciona a los conflictos de identidad que sufre Rios disminuye durante el transcurso de la serie, un período de quince años. Un análisis breve de la última novela de la serie demostrará la evolución del protagonista.

En comparación con The Little Death, la última novela, Rag and Bone, tiene muchos más rasgos culturales chicanos y mexicanos: existe una plétora de referencias a la familia biológica de Rios, aumenta el uso del español, hay muy pocos personajes secundarios que no sean chicanos o latinos y, finalmente, Rios consigue un amante chicano/méxico-americano, Johnny DeLeon (sic). En esta novela, el protagonista ya es un hombre mayor, casi llega a los cincuenta años y está muy consciente de que está envejeciendo. Después de trabajar por tantos años como abogado, Rios ha resuelto una gran cantidad de muertes misteriosas, ha cuidado a un amante enfermo que fallece por SIDA y ha abusado continuamente su cuerpo con el alcohol. Al principio de la novela en cuestión, Rios experimenta un ataque cardiaco y luego pasa mucho tiempo recuperándose. En el núcleo de la narrativa, se encuentra una relación tempestuosa con algunos miembros de su familia, los cuales aparecen en un contexto misterioso: Rios se da cuenta de que tiene una sobrina mayor, Vicky Trujillo, que ya tiene un hijo de diez años, Angel (sic). Resulta ser que su hermana lesbiana, Elena, quedó embarazada cuando era joven y, como quería ser monja, decidió entregarle la bebé a una agencia social para que fuera adoptada. La presencia de Elena, Vicky Trujillo y Johnny DeLeon marca un cambio total en los rasgos culturales de los personajes que comprenden las novelas de Michael Nava.

Revista Iberoamericana, Vol. LXXVI, Núm. 231, Abril-Junio 2010, 409-424
ISSN 2154-4794 (Electrónico) 
Para empezar, DeLeon se convierte en el primero y el único amante de Rios que no es anglosajón. A lo largo de la serie, el abogado mantiene varias relaciones íntimas con hombres anglosajones y una relación duradera con Josh, el amante que fallece por SIDA. La relación entre Rios y DeLeon marca la primera vez que Rios erotiza y forma una relación íntima con un personaje chicano o latino. Es precisamente esta relación la que señala que Rios está superando su conflicto de identidad relacionado con el racismo interno. Johnny DeLeon es un obrero méxicoamerciano que tiene su propio negocio en el campo de la construcción; habla el espanglish y casi llega a ser jugador de béisbol a nivel profesional cuando era joven. De hecho, DeLeon y Rios se parecen mucho: a los dos les fascina el béisbol, son más o menos de la misma edad y tienen la misma herencia cultural. Hasta la estructura de sus nombres es similar -un nombre anglosajón y un apellido en español. En cuanto a sus personalidades, también son muy semejantes: los dos son muy honestos y cariñosos. Rios se siente muy cómodo con DeLeon, como si fueran familiares o mejores amigos. El hecho de que se enamoren demuestra que Rios ha aprendido a aceptarse y amarse a él mismo. Irónicamente, quizás intencionalmente, esto ocurre precisamente después de sufrir su ataque cardiaco, donde técnicamente se muere por casi un minuto. Cuando el cardiólogo lo está resucitando, Rios le hace una pregunta en español acerca de la presencia de su hermana, Elena:

Yes, you died for almost a minute. When you came back, you were asking for her. In Spanish. I speak enough to understand what you wanted, so we tracked her down. That took some doing. She wasn't in you medical power of attorney. I had to call your lawyer to find her. (8)

La muerte físicatemporal de Rios se convierte en un símbolo de la muerte de ciertos aspectos de Rios, en su mayor parte, las características que a él no le gustaban. Después de perder a varios amigos gay anglosajones que fallecieron por SIDA, Rios se queda sin su "familia" alternativa, la cual estaba compuesta únicamente de hombres gay anglosajones. La muerte figurativa sirve para recordarle a Rios sobre su herencia cultural, para dirigir su mirada hacia dentro y para ofrecerle la oportunidad de reencontrarse y reintegrarse a su familia biológica. El hecho de que haya usado el español subconscientemente cuando lo resucitaban es la primera pista que el autor nos da para llegar a la conclusión de que el Henry Rios que resucitaron no es el mismo Henry Rios que murió por casi un minuto.

En la novela How Town, la tercera novela de la serie, Rios confiesa que su uso de español es muy limitado y que cuando intenta usarlo, le cuesta mucho trabajo y siempre tiene problemas con la gramática: "Over the years, I had lost my fluency in Spanish though I could still make myself understood, albeit ungrammatically" (49). En esta novela, cuando intenta usar el español, siempre comete errores que

\footnotetext{
Revista Iberoamericana, Vol. LXXVI, Núm. 231, Abril-Junio 2010, 409-424 ISSN 0034-9631 (Impreso) ISSN 2154-4794 (Electrónico)
} 
dificultan su comunicación con los demás. Por ejemplo, cuando intenta hablar con la mamá hispana de una cliente le dice: "Es muy importante que hablo con su hija...Señorita Soto, por favor, llame su hija y pregunta a ella si quiere hablar con $m e$ " (138). El uso del español gramáticalmente incorrecto en combinación con el uso de itálicas en el texto (hasta la palabra " $m e$ " se marca en itálicas) funcionan como barreras entre Rios y los personajes hispanohablantes de la novela. Aunque pueda comunicarse con ellos más o menos bien, es obvio que el protagonista no se identifica culturalmente con dichas personas.

Por lo tanto, el uso del español por parte de Rios durante su resurrección, en Rag and Bone, es sorprendente y alucinante. Nos sorprende porque parece tan natural y sale de su subconsciente sin tener que hacer un gran esfuerzo. A la vez, revela que Rios, aunque haya pasado tantos años reprimiendo sus raíces culturales, todavía guarda residuos de su herencia cultural en el fondo de su subconsciente. Además, el hecho de que haya preguntado por su hermana, Elena, y no por un amigo ni por su amante difunto, Josh, es una señal que quiere recuperar parte de su historia por medio de su familia biológica, la que mejor representa su herencia cultural, y es lo opuesto de lo que hizo por varios años, cuando pasaba su vida rodeado por hombres anglosajones que formaron parte de su familia gay sustituta. Aunque tenga el corazón frágil, Rios necesita prepararse para empezar una vida nueva. Ahora tiene la oportunidad y, yo diría, la necesidad de crear a una familia un poco más estable donde cada aspecto de su identidad, tanto su sexualidad como su herencia cultural, pueda existir en un espacio emocional seguro y cómodo. Esta vez, su familia sustituta estará compuesta por miembros que son un poco más diversos. DeLeon figura entre los miembros más importantes de la nueva familia.

Después de pasar algunos días recuperándose en el hospital, Rios regresa a su casa. Un día decide caminar a una tienda que queda a unas cinco cuadras de su casa para devolver una película. Para regresar, tiene que subir por una calle inclinada, se cansa mucho y se sienta en el bordillo en frente de una casa. Debido a lo cansado y debilitado que se siente, Rios dice, "At that moment, I would happily have died rather than get up” (43). Aquí es donde entra DeLeon en su vida, pues estaba trabajando en la casa donde se sienta el protagonista y lo rescata de sus pensamientos negativos. Al principio, la energía erótica entre los dos personajes es casi inexistente, por lo menos de parte de Rios. La voz narrativa describe a DeLeon de una manera muy sencilla, sin los adornos que el Rios “difunto” siempre les daba a los hombres anglosajones que le llamaban la atención:

His face came into focus; skin the color of walnuts, short curly reddish-brown hair, a darker goatee flecked with gray. His eyes were narrowed in what appeared to be the permanent squint of someone who spent a lot of time in the sun. When he

Revista Iberoamericana, Vol. LXXVI, Núm. 231, Abril-Junio 2010, 409-424
ISSN 2154-4794 (Electrónico) 
stooped down, I saw they were green. I guessed he was a few years younger than me but slightly more battered; deep lines bracketed his mouth, the flesh sagged beneath his eyes. He was long-legged, with a workingman's heavily muscled arms and chest. A potbelly pressed against his shirt. He grinned as if we were old friends and not two strangers meeting under peculiar circumstances. (43)

En esta descripción de DeLeon, no se encuentra el erotismo que solía marcar los intereses románticos del protagonista. Lo poco que quizás se pueda interpretar como rasgo erótico o exótico (como "green eyes"), se contradice con "the flesh sagged beneath his eyes"; a la vez que "heavily muscled arms and chest" se cancela por el "potbelly pressed against his shirt". El encuentro entre los dos hombres, como lo describe el narrador, es un encuentro entre amigos. No obstante, la cosa más interesante de DeLeon es su herencia cultural. Ofrece llevarlo a su casa en su camión, pero DeLeon, simbólicamente, también es el camino a la casa de la adolescencia de Rios, porque implica la cultura mexicana que marcó la juventud del protagonista. Cada vez que los dos están en un solo espacio, Rios se acuerda de su niñez porque algún objeto despierta un recuerdo reprimido de su herencia cultural. Por ejemplo, cuando Rios está en el camión, ve una imagen de la Virgen de Guadalupe e inmediatamente piensa en su niñez. Durante una cita, DeLeon invita a Rios a comer en un restaurante mexicano, donde Rios reacciona a varios símbolos que aparentemente no había visto en muchos años: los carteles de las películas mexicanas en las paredes y el metate en la mesa. Cuando ve el metate, Rios le dice a DeLeon, "My grandmother had a metate exactly like this one" y DeLeon le responde, "Every 'buelita had that metate” (55). Para Rios, la cita resulta ser un regreso a sus raíces culturales.

Por lo general, Rios y DeLeon comparten una historia muy parecida: sus padres eran casi iguales (violentos y machistas) y a los dos les encantaba jugar al béisbol. A ambos, el béisbol también les hace pensar en su niñez y les permite revivir la relación que tenían con su respectivo padre. Por eso, el hecho de que la segunda cita tome lugar en un partido de béisbol es muy significativo. Al asistir al partido, los dos regresan emocionalmente a los momentos formativos de su pasado. Para Rios, los partidos a que asistía con su padre, son los pocos recuerdos positivos que tiene de él: "Those were the happiest moments I ever had with my dad. By the time I was ten, our expedition to el béisbol were over, and after that there were no happy moments” (67). La cita resulta ser una experiencia muy positiva para Rios y es después de esta escena que el protagonista empieza a desarrollar un sentir cariñoso e íntimo por DeLeon. La relación es tan importante porque DeLeon no es sólo un agente para el reencuentro de Rios con su propia herencia cultural sino también simboliza al padre de Rios. Por ejemplo, cuando regresan a la casa del protagonista después del partido, DeLeon empieza a acercársele físicamente. DeLeon toma la

Revista Iberoamericana, Vol. LXXVI, Núm. 231, Abril-Junio 2010, 409-424
ISSN 2154-4794 (Electrónico) 
mano de Rios en la suya y éste inmediatamente se acuerda de la mano de su padre: "His hand was warm and callused, a workingman's hand. My father's hand" (69). Es precisamente después de este momento íntimo que Rios empieza a sentir una atracción física y emocional por DeLeon: "He was so handsome at that moment-his chin forward defiantly but his eyes unguarded and his hair mashed down from his baseball cap. I felt a surge of tenderness and affection for him” (71).

Así, DeLeon se convierte en miembro de la familia sustituta que Rios va formando, una familia que, esta vez, se compone sólo de chicanos y que incluye a algunos miembros que no se identifican como gay. Los otros miembros son Elena, Vicky y Angel -todos relacionados con Rios biológicamente. Para incorporar a Vicky y a Angel, Rios necesita continuar su lucha contra la homofobia. Vicky fue criada dentro del barrio y tiene rasgos de una "chola” típica; representa la cultura chicana y la de los pandilleros. Ahora, ella se ha convertido en una evangelista y cree que la homosexualidad es una aberración. De esta forma, Vicky sirve como emblema de las instituciones religiosas que se oponen a los derechos para los homosexuales. Desde que Vicky conoce a su tío por primera vez, le deja saber su posición sobre la homosexualidad: "Jesus got me off drugs. Now He wants Pete and me and Angel to be a family, a Christian family. I know you're not a Christian. I know what you are” (81). Existe mucha tensión entre Rios y Vicky, sin embargo, como están relacionados por sangre, tienen que apoyarse y aguantarse. Vicky aguanta a Rios porque lo necesita como abogado. Rios aguanta a Vicky porque le está haciendo un favor a Elena; entiende que su hermana está intentando mejorar la relación entre ella y su hija después de haberla abandonado cuando era bebé. Por otro lado, a Rios le interesa mucho el desarrollo educativo y emocional del hijo de Vicky, porque en Angel se ve a él mismo cuando era niño y lo quiere rescatar de un ambiente aparentemente dañino. En realidad, la relación que se desarrolla entre Rios y Angel es una relación de padre e hijo. Vicky intenta interferir en la dinámica entre su hijo y el tío al advertirle a Angel que su tío es homosexual. Cuando Rios se da cuenta de que Vicky hace esto, le dice: "Stop scaring Angel about me. I'm not going to hurt him” (86). Para proteger el espacio emocional que ha creado en su casa, un ambiente seguro donde no existían amenazas homofóbicas, insiste en que Vicky cambie su vocabulario y que lo acepte como parte de la familia porque están relacionados biológicamente: “And I won't hear the word joto in my house again. Or maricón or any other gutter words you've taught him to use about people like me and your mother. Remember something, Vicky, our blood flows through your veins. Whatever we are, you and Angel carry inside you” (86). De tal manera, Rios logra alterar el discurso homofóbico predominante en la cultura chicana. Vicky, como representante del "barrio" chicano, es forzada a participar en un diálogo sobre la homosexualidad y es obligada a aceptar a los miembros de su familia que son gay: Rios y Elena.

Revista Iberoamericana, Vol. LXXVI, Núm. 231, Abril-Junio 2010, 409-424
ISSN 2154-4794 (Electrónico) 
La tensión entre Rios y Vicky persiste a lo largo de la narrativa. No obstante, se soportan y llegan a respetarse el uno al otro, hasta crear una familia más o menos estable. Vicky es arrestada por el asesinato de su esposo y Rios sirve como su abogado. Irónicamente, a Rios le conviene mucho que esté encarcelada porque le da a él la oportunidad de pasar tiempo creando la familia que de verdad quiere: DeLeon, como su pareja, y Angel, como su hijo. Estando Vicky en la cárcel, Rios puede proteger a Angel del ambiente homofóbico que su mamá mantiene. A la vez, puede actuar como el padre sustituto de su sobrino. La relación entre Rios, DeLeon y Angel florece y establecen una rutina familiar que incluye el asistir a partidos de béisbol, el comer juntos y la participación de los tres en otras actividades de recreo.

Además de convertirse en miembro de la familia, DeLeon participa en la lucha de Rios contra la homofobia. DeLeon le explica a Rios que no permite que sus empleados hagan comentarios homofóbicos: "We do work for gay guys all the time, and when we do, I tell my crew if I hear any fag jokes of any kind or remarks like that, they're gone. I tell them, these people are feeding your families, you should show some courtesy" (95). Por otro lado, DeLeon es el que intenta inculcar en Rios la idea de que es posible ser chicano/mexicano y gay. Para el Rios de antes del ataque cardiaco, ser gay y ser chicano/mexicano era una dicotomía; no era posible integrar esos dos aspectos de su identidad, sólo podía ser uno o el otro y por varios años decidió vivir como hombre gay con algunos rasgos anglosajones. Como explica DeLeon, en la cultura chicana/mexicana, la idea de ser gay siempre llevaba sus connotaciones y estereotipos negativos:

Look, Henry, you're mexicano, too, so you know the drill. Men are men. The only homosexual Mexican I ever met when I was a kid was one of my grown-up cousins who lived with his mom and wore more makeup than her. That's what I thought all homosexuals were like. (70)

Para ambos, vivir como hombre gay significaba tener que rechazar su masculinidad porque su herencia cultural no permitía la existencia de un hombre gay sin relegarlo a un espacio femenino. El hombre gay se contrastaba completamente con la imagen del macho típico. Como sugiere Ilán Stavans en su ensayo "The Latin Phallus” (1996), el macho mexicano siempre se portaba como un hombre activo y dominante mientras que los homosexuales se percibían como pasivos y vulnerables:

In the Mexico of the seventies in which I grew up, common sense had it that machos were the unchaste victims of an unsurpassed inferiority complex. Unchaste victims-impure, yes, but sympathetic characters and commanding figures.

Revista Iberoamericana, Vol. LXXVI, Núm. 231, Abril-Junio 2010, 409-424
ISSN 2154-4794 (Electrónico) 
Homosexuals, on the other hand, were considered oversensitive, vulnerable, unproved in the art of daily survival. At school, the boys were constantly made to taste their muscular strength. (155)

Es claro que para DeLeon existe la problemática de no poder identificarse como un hombre gay moderno, especialmente con respecto a la cultura gay anglosajona. Mientras Rios ha pasado muchos años sumergido exclusivamente en un mundo gay anglosajón, DeLeon sólo ha practicado actos homosexuales de vez en cuando y se identifica como bisexual. Para ambos, el negociar un espacio donde su sexualidad y su herencia cultural puedan coexistir en paz, requiere el cambio total de la construcción ideológica que les ha formado con respecto al estilo de vida de los hombres gay. En su ensayo, "Chicano Men: A Cartography of Homosexual Identity and Behavior" (1991), Tomás Almaguer propone que para el sujeto chicano tal proceso se lleva a cabo de una manera muy distinta al del hombre anglosajón:

Chicanos, as well as other racial minorities, do not negotiate the acceptance of a gay identity in exactly the same way white American men do. The ambivalence of Chicanos vis-à-vis a gay sexual identity and their attendant uneasiness with white gay/lesbian culture do not necessarily reflect a denial of homosexuality. Rather, I would argue, the slow pace at which this identity formation has taken root among Chicanos is attributable to cultural and structural factors which differentiate the experiences of the white and non-white populations in the U.S. (86)

Entonces, DeLeon y Rios representan una alternativa al "chicano/mexicano homosexual” estereotipado que describe Stavans. En la narrativa, su masculinidad es subrayada por los rasgos masculinos de las descripciones de sus cuerpos, la ropa que usan y la comida que comen. Asimismo, la importancia del béisbol y la recuperación de su herencia cultural los convierte en hombres gay indistintamente chicanos/méxico-americanos y masculinos.

Para el final de la novela, Rios y DeLeon han podido convencer a Elena y a Vicky de que sirven como buenos ejemplos para Angel. Los dos están totalmente involucrados en el desarrollo educativo y emocional del niño, y Rios se encuentra muy contento con su familia sustituta. Elena y Vicky se ven frecuentemente ahora que ésta ha salido de la cárcel; Vicky y Angel viven en un apartamento cerca de la casa del tío; Rios y DeLeon ven a Angel diariamente, criándolo como si fuera su propio hijo. La última escena de la novela, y de la serie, revive la juventud de Rios y propone la salida de los estereotipos de una familia tradicional; Rios, como el padre de esta familia, rompe el círculo de homofobia que caracterizaba sus experiencias durante la adolescencia: llega a casa después del trabajo y encuentra

Revista Iberoamericana, Vol. LXXVI, Núm. 231, Abril-Junio 2010, 409-424
ISSN 2154-4794 (Electrónico) 
a Angel haciendo su tarea enfrente del televisor, viendo un partido de béisbol, por supuesto.

Rios logra, entonces, distanciarse de los abusos físicos y emocionales de su adolescencia y consigue crear un espacio queer como el que Cherríe Moraga pide en su ensayo “Queer Aztlán: The Re-formation of Chicano Tribe” (1993): un espacio donde los chicanos gay y las chicanas lesbianas puedan vivir en paz sin ser víctimas de la homofobia presente en la cultura chicana. Moraga insiste en que un "Queer Aztlán” se establezca dentro de un espacio cultural chicano:

Chicana lesbians and gay men do not merely seek inclusion in the Chicano nation; we seek a nation strong enough to embrace a full range of racial diversities, human sexualities, and expressions of gender. We seek a culture that can allow for the natural expression of our femaleness and maleness and our love without prejudice or punishment. In a “queer” Aztlán, there would be no freaks, no “others” to point one’s finger at. (164)

Como he señalado, Henry Rios experimenta varias transformaciones a lo largo de la serieque se llevan a cabo durante su lucha contra dos fuerzas poderosas: la homofobia y el racismo. Mientras Rios conscientementelucha contra la homofobia en los espacios públicos que habita y lidia con los discursos que encuentra allí, subconscientemente lucha contra su propia homofobia y los valores culturales asimilados dentro de una cultura homofóbica. Asimismo, mientras lucha concientemente contra un ambiente racista, tiene que enfrentar su propio racismo buscando estrategias para salir del círculo que repite la homofobia a nivel individual y colectivo. El miedo que surge por la amenaza de ser rechazado de su propia cultura y de la cultura dominante afecta la identidad del sujeto, lo cual puede dejarlo como un náufrago cultural, en una especie de "tierra de nadie". Después de sentirse amenazado por no seguir las normas de la sociedad dominante anglosajona, el sujeto reprime y rechaza los aspectos de su identidad que se perciben como inaceptables para la participación en dicha sociedad. Una vez eliminadas las características inaceptables, participa legítimamente en la sociedad, pues intenta adoptar las normas físicas, culturales e ideológicas que se alaban en la cultura dominante. Como explica Gloria Anzaldúa en Borderlands/La Frontera: The New Mestiza (1987):

We're afraid of being abandoned by the mother culture, la Raza, for being unacceptable, faulty, damaged. Most of us unconsciously believe that if we reveal this unacceptable aspect of the self our mother / culture / race will totally reject us. To avoid rejection, some of us conform to the values of the culture, push the unacceptable parts into the shadows. (20)

Revista Iberoamericana, Vol. LXXVI, Núm. 231, Abril-Junio 2010, 409-424
ISSN 2154-4794 (Electrónico) 
La teoría deAnzaldúa ofrece una posible explicación para el comportamiento del personaje Henry Rios. El miedo que experimenta por la amenaza de ser abandonado o rechazado de su propia cultura (y no se debe pasar por alto el miedo de ser víctima de la violencia) lo ubica en una posición marginal. Su búsqueda se basa en el deseo de centrarse en un espacio donde él, y consiguientemente otras personas como él, puedan existir sin ser víctimas de la homofobia ni del racismo. En su intento de obtener dicho estatus, Rios reprime algunos aspectos de su identidad para poder destacar otros aspectos necesarios para adelantar su compromiso social. Por eso, la lucha contra la homofobia al inicio de la serie se lleva a cabo en un contexto cultural anglosajón. Después de combatir la homofobia en un contexto anglosajón, Rios intenta luchar contra la homofobia en la cultura chicana y cambiar dicho espacio cultural para que pueda ser un hombre chicano y gay sin ser castigado por la cultura chicana ni por la cultura dominante anglosajona. El protagonista realiza la construcción de un espacio seguro donde él y otras personas como él pueden superar las amenazas de una sociedad homofóbica y racista.

\section{BiBLIOGRAFÍA}

Almaguer, Tomás. "Chicano Men: A Cartography of Homosexual Identity and Behavior”. Differences 3/2 (1991): 75-100.

Anzaldúa, Gloria. Borderlands/La frontera: The New Mestiza. San Francisco: Aunt Lute Books, 1987.

Dyer, Richard. White. Nueva York: Routledge, 1997.

Fanon, Frantz. Black Skin/White Masks. Nueva York: Grove Press, 1967.

Foster, David William. El ambiente nuestro: Chicano/Latino Homoerotic Writing. Tempe: Bilingual Press/Editorial Bilingüe, 2006.

Lacan, Jacques. Ecrits: A Selection. Nueva York: W.W. Norton, 1977.

Levine, Martin P. Gay Macho: The Life and Death of the Homosexual Clone. Nueva York: New York UP, 1998.

Moraga, Cherríe. “Queer Aztlán: The Re-formation of Chicano Tribe”. The Last Generation. Boston: South End Press, 1993.

Nava, Michael. How Town. Nueva York: Harper and Row, 1990. The Little Death. Los Angeles: Alyson Books, 2003. Rag and Bone. Nueva York: Berkley Prime Crime, 2002.

Pérez, Emma. The Decolonial Imaginary: Writing Chicanas into History. Bloomington: Indiana UP, 1999.

Signorile, Michelangelo. Life Outside: The Signorile Report on Gay Men. Nueva York: HarperPerennial, 1997. 
Stavans, Ilán. "The Latin Phallus". Muy Macho: Latino Men Confront Their Manhood. Ray González, ed. Nueva York: Anchor Books, 1996. 143-64.

Stoler, Anne. Race and the Education of Desire. Durham: Duke UP, 1995.

ISSN 0034-9631 (Impreso) 